\title{
SINGULAR FUNCTIONS ASSOCIATED WITH MARKOV CHAINS ${ }^{1}$
}

\author{
JOHN R. KINNEY ${ }^{2}$
}

We suppose $\left\{x_{i}\right\}, i=1,2, \cdots$ to be a chain with a finite number of states, $0,1, \cdots, M-1$, and consider the random variable $X$ $=\sum_{i=1}^{\infty} x_{i} M^{-i}$ and its associated distribution function $F(x)$ $=\operatorname{Prob}\{X<x\}$. We write $F(A)=\operatorname{Prob}\{X \in A\}=\int_{A} d F(x) . F(A)$ is a completely additive probability measure on the Borel field of sets in $[0,1]$ generated by sets of the form $\{F(x)<\alpha\}$. Harris [1] has shown that under very general conditions on the stationarity of the chain that $F(x)$ is a purely singular function and that $\phi(t)_{t \rightarrow 0} \rightarrow 0$ where $\phi(t)$ is the Fourier-Stieltjes transform $\phi(t)=\int e^{i t x} d F(x)$. Wiener and Wintner [2] used the connection between the Lipschitz condition satisfied by $F(x)$ and the behavior of $\phi(t)$ to show that there are purely singular functions $F(x)$ for which $\phi(t)_{t \rightarrow \infty}=O\left(t^{-\alpha}\right)$ for all $\alpha<1 / 2$.

Salem [3] showed the connection between the Hausdorff measure of the set $E$ on which $F(A)$ is concentrated and the behavior of $\phi(t)$ for large $t$. Although in our case $\phi(t)_{t \rightarrow \infty} \rightarrow 0$, the Lipschitz condition and the Hausdorff dimension of $E$ still play a role. Namely, when the $x_{i}$ form a stationary Markov chain, with a single ergodic class, they are the entropy, in the sense of Shannon [4], of the sequence $\left\{x_{i}\right\}$ considered as the sequence of states of a symbol-generating source.

The dimensional number $\beta(E)$ of a set $E \subset[0,1]$ is defined as follows: If $\mu \geqq \max _{i}\left|I_{i}\right|$, where $\left\{I_{i}\right\}$ is a set of intervals, and $E \subset \cup I_{i}$, we say $C \mu=U I_{i}$ is a covering of $E$ of norm $\mu$. We let

$$
\Gamma(\gamma, C \mu, E)=\sum\left|I_{i}\right|^{\gamma} \text {. }
$$

The $\gamma$-dimensional Hausdorff measure of $E$ is

$$
\Gamma(\gamma, E)=\lim _{\mu \rightarrow 0} \text { g.l.b. } \Gamma(\gamma, C \mu, E)
$$

where the greatest lower bound is taken over all coverings of norm $\mu$. $\beta(E)$ is the number such that, for all $\epsilon>0$,

$$
\Gamma[\beta(E)-\epsilon, E]=\infty, \quad \Gamma[\beta(E)+\epsilon, E]=0 .
$$

We suppose then the $\left\{x_{i}\right\} i=1,2, \cdots$, to be a Markov chain,

Received by the editors August 12, 1957, and in revised form March 19, 1958.

1 The research in this document was supported jointly by the Army, Navy and Air Force under contract with the Massachusetts Institute of Technology.

${ }^{2}$ Staff Member, Lincoln Laboratory, Massachusetts Institute of Technology. 
with stationary transition probabilities with matrix $\left\|a_{i j}\right\|$, a single ergodic class, initial probabilities $M^{-1}$, and stationary probabilities $b_{i}$.

We let $\alpha=-\sum_{i, j} b_{i} a_{i j} \log _{M} a_{i j}$, a number proportional to the entropy in the sense of Shannon [4].

Theorem 1. There is a set $E \subset[0,1]$ such that

(1) $F(E)=1$,

(2) $\beta(E)=\alpha$,

(3) $x \in E, \epsilon>0$ imply

$$
\begin{aligned}
& \lim _{h \rightarrow 0} F(x-h, x+h) h^{-\alpha+\epsilon} \rightarrow 0, \\
& \lim _{h \rightarrow 0} F(x-h, x+h) h^{-\alpha-\epsilon} \rightarrow \infty .
\end{aligned}
$$

We let $I(n, k)=\left[k M^{-n},(k+1) M^{-n}\right], I(n, x)$ be that $I(n, k)$ which contains $x$. If $k=\sum_{i=0}^{n-1} k_{n-i} M^{i}$ is the expansion of $k$ in the base $M$, $F[I(n, x)]=M^{-1} \prod_{i=2}^{n} a_{k_{i-1}, k_{i}}$. By the ergodic properties of the chain, the number of times $\left(k_{i-1}, k_{i}\right)=(j, k)$ is $b_{j} a_{j k}[n+O(I)]$ [4]. Hence

$$
F[I(n, x)]=\prod_{i j} a_{i j}^{[n+O(1)] b_{i} a_{i j}}=M^{-[n+O(1)] \alpha}
$$

except for a set of zero measure, which we delete to form $E^{*}, F\left(E^{*}\right)$ $=1$. On $E^{*}$, (3.b) is satisfied.

Although (1) immediately implies (3b), its lack of symmetry does not allow us to conclude that (3a) holds for all points of $E^{*}$. We eliminate the points affected by this lack of symmetry to form a set $E \subset E^{*}$ for which (3a) holds and $F(E)=1$. We proceed as follows: We let $C(\epsilon)$ be those $x$ for which

$$
F[I(n, x)] \geqq|I(n, x)|^{\alpha-\epsilon}
$$

for an infinite number of $n$. Since $C(\epsilon) \subset c E^{*}, F[C(\epsilon)]=0$. We choose the covering $C_{n}(\epsilon)$ of $C(\epsilon)$ by assigning to each $x \in C(\epsilon)$ that interval $I(m, x)$ for which (2) holds for the smallest $m>n$. Thus, the interval assigned to $x$ in the $n$th covering includes the interval assigned to $x$ in any higher covering. Hence, $C_{n}(\epsilon) \supset C_{n+s}(\epsilon)$ in the sense of set inclusion. Also, $C_{n}(\epsilon) \downarrow C(\epsilon)$. From the complete additivity of $F(A)$, $\lim _{n \rightarrow \infty} F\left[C_{n}(\epsilon)\right]=0$. For each $C_{n}(\epsilon)$ we construct a $C_{n}(r, \epsilon)$ and $C_{n}(l, \boldsymbol{\epsilon})$ as follows: If $I(s, k) \in C_{n}(\boldsymbol{\epsilon})$ we assign $I(s, k+1)$ to $C_{n}(r, \epsilon)$, $I(s, k-1)$ to $C_{n}(l, \epsilon)$. We let $D_{n}(\boldsymbol{\epsilon})=C_{n}(\boldsymbol{\epsilon}) \cup C_{n}(r, \epsilon) \cup C_{n}(l, \epsilon)$. If $I(s, k+1) \in C_{n}(r, \epsilon)$ and $I(s, k+1) \subset c C_{n}(\boldsymbol{\epsilon})$, (2) does not hold and hence $F[I(s, k+1)]<|I(n, k+1)|^{\alpha-\epsilon}=|I(n, k)|^{\alpha-\epsilon} \leqq F[I(s, k)]$. Hence,

$F\left[C_{n}(r, \epsilon)\right]=F\left[C_{n}(r, \epsilon) \cap C_{n}(\epsilon)\right]+F\left[C_{n}(r, \epsilon) \cap c C_{n}(\epsilon)\right] \leqq 2 F\left[C_{n}(\epsilon)\right]$. 
Similarly, $F\left[C_{n}(l, \epsilon)\right] \leqq 2 F\left[C_{n}(\epsilon)\right]$. So $F\left[D_{n}(\epsilon)\right] \leqq F\left[C_{n}(\epsilon)\right]$ $+F\left[C_{n}(r, \epsilon)\right]+F\left[C_{n}(l, \epsilon)\right] \leqq 5 F\left[C_{n}(\boldsymbol{\epsilon})\right]$. We note that $D_{n}(\boldsymbol{\epsilon})>D_{n+8}(\boldsymbol{\epsilon})$ and let $D(\epsilon)=\lim _{n \rightarrow \infty} D_{n}(\epsilon) . F[D(\epsilon)] \leqq 5 \lim F\left[C_{n}(\epsilon)\right]=0$. We obtain $E$ by deleting from $E^{*}$ all points belonging to $D\left(\epsilon_{n}\right)$ for a sequence $\epsilon_{n} \downarrow 0$. Having eliminated only a countable number of null sets, we still have $F[E]=1$. However, for $x \in E$, for any $\epsilon$, we can choose $m$ and $k$ so large that if $x \in I(m, j), F[I(m, j+i)]<M^{-m(\alpha-\epsilon)}$ for $i=-1$, 0 , 1. Hence, for $h<M^{-n}, F(x-h, x+h)<3 M^{-n(\alpha-\epsilon)}$ from which we may deduce (3a).

We cover $E$ by a set $C_{n}=\left\{I_{j}\right\}$ of norm $M^{-n}$, by assigning for each $x$ the $I(m, x)$ for which $F[I(m, x)]>|I(m, x)|^{\alpha+\epsilon}$ for the smallest $m \geqq n$. The $I_{j}$ are disjoint, since none is included in another and they cannot overlap. Since $E \subset U_{j} I_{j}, 1 \geqq \sum F\left(I_{j}\right)>\sum\left|I_{j}\right|^{\alpha+\epsilon}$. Hence $\Gamma(\alpha+\epsilon, E) \leqq 1$ for all $\epsilon>0$, so $\beta(E) \leqq \alpha$.

By choosing $h_{0}$ sufficiently small, we can, for any fixed $\epsilon$, find a subset $A\left(h_{0}\right)$ of $E$ such that $F\left[A\left(h_{0}\right)\right]>0.5$, and, for $x \in A\left(h_{0}\right)$, $h \leqq h_{0}$ we have $F(x-h, x+h)<(2 h)^{\alpha-\epsilon}$. We choose any covering $C_{h_{0}}=\mathrm{U} I_{j}$. To each $I_{i}$ we choose a point $x_{i} \in A\left(h_{0}\right)$ and take $I_{i}^{\prime}$ to be the smallest interval containing $I_{i}$ symmetric about $x_{i}$. We note that $\left|I_{i}^{\prime}\right|<2\left|I_{i}\right|$. We then have

$$
\sum\left(2\left|I_{i}\right|\right)^{\alpha-\epsilon} \geqq \sum\left|I_{i}^{\prime}\right|^{\alpha-\epsilon}>\sum F\left(I_{i}^{\prime}\right) \geqq F\left[A\left(h_{0}\right)\right]>0.5 .
$$

So, for $\epsilon>0, \Gamma\left[\alpha-\epsilon, C_{h_{0}}, A\left(h_{0}\right)\right]>0.5$. Since $C_{h_{0}}$ was an arbitrary covering of $A\left(h_{0}\right)$ of norm $h_{0}, \Gamma\left[\alpha-\epsilon, A\left(h_{0}\right)\right]>0.5$. Hence $\beta\left[A\left(h_{0}\right)\right]$ $\geqq \alpha$. Since $E \supset A\left(h_{0}\right), \beta(E) \geqq \alpha$. Hence (2) holds. This establishes our theorem.

An example: H. G. Eggleston [5] has shown that the set

$$
S=\left\{x \mid \lim _{n \rightarrow \infty} \sum_{i=1}^{n} x_{i} / n \leqq a \leqq(M-1) / 2\right\} \text { has } \beta(S)=\alpha,
$$

where $M^{\alpha}=\left[k r^{a}\right]^{-1}, k=(1-r) /\left(1-r^{M}\right)$, for $r$ the positive real root of $\sum_{i=0}^{M-1}(i-a) r^{i}=0$. He proves this by showing $\alpha \geqq \beta\left(S^{*}\right) \geqq \beta\left(S_{*}\right) \geqq \alpha$, where $S^{*}, S_{*}$ are obtained by replacing the limit in the definition of $S$ by limit inferior and limit superior and using rather sophisticated methods to obtain his coverings.

We give a proof using the point of view of information theory. We let $x_{j}=i$, Prob $p_{i}, i=0, \cdots, M-1$, independently of $j$, subject to the restrictions $E\left(x_{j}\right)=\sum i p_{i}=a, \sum p_{i}=1$. We vary the probabilities $p_{i}$ so as to maximize the entropy $H(X)=-\sum p_{i} \log _{M} p_{i}$, of the sequence $\left\{x_{i}\right\}$ considered as a source, subject to the given side conditions. We find the maximum to be given by $k r^{i}$. Our restrictions imply $k \sum_{i=0}^{M-1} r^{i}=1, \quad k \sum_{i=0}^{M-1} i r^{i}=a$. This yields $k=\left[\sum_{0}^{M-1} r^{i}\right]^{-1}$ 
$=1-r / 1-r^{M} . \sum_{i=0}^{M-1}(i-a) r^{i}=0$. We have also

$$
\begin{aligned}
H(X) & =-\sum_{0}^{M-1} k r^{i} \log _{M} k r^{i}=-\sum k r^{i} \log _{M} k-\sum k i r^{i} \log _{M} r \\
& =-\log _{M} k-a \log _{M} r=-\log _{M} k r^{a},
\end{aligned}
$$

so $H(X)=\alpha$. However, our measure $F(A)$ imposed on the interval $[0,1]$ by this assignment of probabilities, applied to intervals $I(n, x)$ corresponding to the partial sums of $x$, gives

$$
F[I(n, x)]=k^{n} r^{\Sigma_{i=1}^{n} x_{i}}
$$

so the set $S_{0}$ which corresponds to the set $E$ of Theorem 1 is

$$
S_{0}=\left\{x \mid \lim _{n \rightarrow \infty} \sum_{i=1}^{n} x_{i} / n=a\right\} \text {, and, by Theorem } 1, \beta\left(S_{0}\right)=\alpha .
$$

We note that, in terms of $F(A), S^{*}$ is the set

$$
\begin{aligned}
& S^{*}=\{x \mid F[I(n, x)] \\
& \left.\quad=k^{n} r^{\Sigma^{n} x_{i}} \geqq M^{-n \alpha}=|I(n, x)|^{\alpha} \text { for infinitely many } n\right\} .
\end{aligned}
$$

We construct the covering $C_{N}$ of $S^{*}$ of norm $M^{-N}$ by assigning to each $x \in S^{*}$ that interval $I(m, x)$ for which, for the smallest $m>N$, $F[I(m, x)] \geqq M^{-m \alpha}=|I(m, x)|^{\alpha}$. The $I(m, x)$ are disjoint, so

$$
1 \geqq F\left(C_{N}\right)=\sum F[I(m, x)]>\sum|I(m, x)|^{\alpha}=\Gamma\left(\alpha, C_{N}, S^{*}\right) .
$$

Hence, $\Gamma\left(\alpha, S^{*}\right) \leqq 1$, so $\beta\left(S^{*}\right) \leqq \alpha$. Since $S^{*} \supset S \supset S_{0}, \alpha \geqq \beta\left(S^{*}\right)$ $\geqq \beta(S) \geqq \beta\left(S_{0}\right)=\alpha$. So $\beta(S)=\alpha$.

We suppose ourselves restricted to sending two-state $[0,1]$ pulses in the transmission of messages. We can then take symbols $\left\{y_{i}, i=1, \cdots, \mu\right\}$ to be binary numbers of lengths respectively $t_{i}$. A message will be a sequence of the $y_{i}{ }^{\prime}$ s. $Z_{j}$, the $j$ th symbol, will be a function whose range are the $y$ 's. An infinite message will be made to correspond to a point $y$ in $[0,1]$ by the dyadic expansion

$$
Y=\sum Z_{k} 2^{-\Sigma_{i-1}^{k} t_{i}}
$$

The set of such points we will call the message set, $Q$. We assume restrictions on the choice of the $y_{i}$ to have been made so that two different messages cannot correspond to the same point. This will imply that different finite messages of the same length will correspond to disjoint dyadic intervals of the line. 
Each finite message of length $n$ will correspond to an interval in $[0,1]$ from the $n$th dyadic net. We suppose $t_{j}=M$ to be the length of the longest symbol. We let $N(t)$ be the number of finite messages of length $t$. It is clear that we can cover the message set $Q$ by $N(t)$ intervals of length $2^{-t}, N(t+1)$ intervals of length $2^{-(t+1)}, \cdots$, $N(t+M)$ intervals of length $2^{-(t+M)}$. These may, of course, involve overlapping; however, we have an approximation

$$
\Gamma\left[\gamma, C\left(2^{-t}\right), Q\right] \leqq \sum_{0}^{M} N(t+i) 2^{-(t+i) \gamma}
$$

to the Hausdorff $\gamma$-dimensional measure of the set $Q$. We let $k(j)$ be the number of symbols of length $j$. As Shannon shows [1], $N(t)$ satisfies the difference equation

$$
N(t)=\sum_{1}^{M} k(j) N(t-j)
$$

so that $N(t)$ is asymptotically approximated by $k \lambda^{t}$ where $\lambda$ is the largest root of

$$
\lambda^{t}=\sum_{1}^{M} k(j) \lambda^{t-j}
$$

Hence,

$$
\Gamma\left[\gamma, C\left(2^{-t}\right), Q\right] \leqq K \lambda^{t} 2^{-\gamma t}
$$

for large $t$. For $\gamma>\log _{2} \lambda$,

$$
\Gamma\left[\gamma, C\left(2^{-t}\right), Q\right] \rightarrow 0
$$

so $\beta(Q) \leqq \log _{2} \lambda$.

We propose to send $y_{j}$ independently of what has been sent before with probability $p_{j}=\lambda^{-t_{j}}$. By [3], $\sum p_{j}=1$. On each of our intervals of length $2^{-t}$ corresponding to messages of length exactly $t$, the increase of $F(x)$, the distribution imposed on $[0,1]$ by our mapping, and by our choice of message distribution will be $\lambda^{-t}$. Hence, on $Q$, our function $F(x)$ satisfies a Lipschitz condition $\operatorname{Lip} \log _{2} \lambda$, and no weaker Lipschitz condition. Since $Q$ consists of the points of increase of $F(x), Q$ is closed. Hence we may cite the theorem of J. Gillis [6], to the effect that if $F(x)$ continuous and monotone takes its increase on a closed set $E$, satisfies a Lipschitz condition of no smaller order than Lip $\delta$, then the Hausdorff $\delta$-dimensional measure of $E$ is positive. Hence $\beta(Q) \geqq \log _{2} \lambda$. 
Thus, if we let $C$ denote the capacity of a channel in the sense of Shannon, we have, for the case described above.

Theorem 2. $\beta(Q)=C$.

\section{REFERENCES}

1. T. E. Harris, On chains of infinite order, Pacific J. Math. vol. 5 (1955) pp. $707-$ 724.

2. N. Wiener and A. Wintner, Fourier-Stieltjes transform and singular finite convolutions, Amer. J. Math. vol. 60 (1938) pp. 513-522.

3. R. Salem, On singular monotone functions whose spectrum has a given Hausdorff dimension, Ark. Mat. vol. 1 (1952) pp. 353-356.

4. C. Shannon, A mathematical theory of communication, Bell System Technical Journal vol. 27 (1948) pp. 379-423.

5. H. G. Eggleston, Sets of fractional dimensions which occur in some problems of number theory, Proc. London Math. Soc. vol. 54 (1953) pp. 42-93.

6. J. Gillis, $A$ note on a theorem of Myrberg, Proc. Cambridge Philos. Soc. vol. 33 (1937) pp. 419-424.

Massachusetts Institute of Technology 